\title{
Guide pratique pour estimer la dose interne à partir des résultats de mesure de surveillance (projet IDEAS)
}

\author{
H. DOERFEL ${ }^{1}$, A. ANDRASI ${ }^{2}$, I. AUBINEAU-LANIÈCE ${ }^{3}$, M. BAILEY ${ }^{4}$, \\ V. BERKOVSKI ${ }^{5}$, E. BLANCHARDON ${ }^{3}$, C.-M. CASTELLANI $^{6}$, C. HURTGEN ${ }^{7}$, \\ J.-R. JOURDAIN ${ }^{3}$, B. LE GUEN ${ }^{8}$, I. MÁLÁTOVA 9 , J. MARSH ${ }^{4}$, M. PUNCHER ${ }^{4}$
}

(Manuscrit reçu le 16 août 2004, accepté le 3 octobre 2004)

RÉSUMÉ Au cours des exercices récents d'intercomparaison, les estimations de dose interne et d'incorporation calculées par les différents participants à partir des données de surveillance ont montré une grande variabilité. Ceci est dû principalement à la diversité des méthodes et des hypothèses appliquées dans la procédure d'évaluation. À partir de ce constat, le besoin d'une harmonisation des procédures a été formulé à travers un projet de recherche européen dans le cadre du $5^{\mathrm{e}}$ PCRD. Le but de ce projet, IDEAS, est de proposer un guide pratique pour homogénéiser les évaluations de dose interne et d'incorporation. Le projet IDEAS a débuté en octobre 2001 et s'achèvera en mai 2005. Huit institutions de sept pays européens y participent. Il est aussi fait appel aux contributions de professionnels de la dosimétrie interne de toute l'Europe pour assurer un large consensus au résultat du projet. IDEAS s'intéresse de près à certains des objectifs du comité 2 de la CIPR et les deux groupes entretiennent une étroite collaboration depuis 2003. Afin de s'assurer que les conseils soient applicables à un large spectre de situations concrètes, la première étape a été la compilation d'une base de données bien documentée de cas de contamination interne. En parallèle, une version améliorée d'un logiciel déjà existant a été développée et distribuée aux partenaires pour une utilisation dans la suite du projet. Un grand nombre des cas de la base de données a été évalué indépendamment par les partenaires du projet en utilisant le même logiciel et les résultats ont été analysés. Sur la base de ces évaluations, une première version du guide a été éditée et discutée avec les professionnels de la dosimétrie du monde entier par le biais d'un séminaire virtuel sur Internet en juin 2004. Les indications seront révisées et affinées à partir des discussions et expériences rapportées au cours de ce séminaire virtuel ainsi que du résultat de l'exercice d'intercomparaison qui sera organisé dans le cadre du projet et ouvert à tous les professionnels de la dosimétrie interne.

\footnotetext{
Forschungszentrum Karlsruhe GmbH, P.O. Box 3640, 76021 Karlsruhe, Allemagne.

KFKI Atomic Energy Research Institute, 1525 Budapest 114, P.O. Box 49, Hongrie.

IRSN, LEDI, B.P. 17, 92262, Fontenay-aux-Roses Cedex, France.

National Radiological Protection Board, Chilton, Didcot, Oxon, OX11 ORQ, Royaume-Uni.

Radiation Protection Institute, Melnikova 53, 04050 Kiev, Ukraine.

ENEA Radiation Protection Institute, 16 via dei Colli, 40136 Bologna, Italie.

SCK • CEN, Boeretang 200, $2400 \mathrm{Mol}$, Belgique.

Électricite de France, SCAST-LAM, 6 rue Ampère, 93203 Saint-Denis Cedex, France.

National Radiation Protection Institute, Srobarova 48, 10000 Praha 10, République Tchèque.
} 


\begin{abstract}
General guidelines for the assessment of internal dose from monitoring data (project IDEAS).

In recent major international intercomparison exercises on intake and internal dose assessments from monitoring data, the results calculated by different participants varied significantly. This was mainly due to the broad variety of methods and assumptions applied in the assessment procedure. Based on these experiences the need for harmonisation of the procedures has been formulated within an EU research project under the 5th Framework Programme. The aim of the project, IDEAS, is to develop general guidelines for standardising assessments of intakes and internal doses. The IDEAS project started in October 2001 and will end in March 2005. Eight institutions from seven European countries are participating. Inputs from internal dosimetry professionals from across Europe are also being used to ensure a broad consensus in the outcome of the project. The IDEAS project is closely related to some goals of the work of committee 2 of the ICRP and since 2003 there has been close cooperation between the two groups. To ensure that the guidelines are applicable to a wide range of practical situations, the first step has been to compile a database of well-documented cases of internal contamination. In parallel, an improved version of an existing software package has been developed and distributed to the partners for further use. A large number of cases from the database have been evaluated independently by partners in the project using the same software and the results have been reviewed. Based on these evaluations, guidelines are being drafted and will be discussed with dosimetry professionals from around the world by means of a virtual workshop on the Internet early in 2004. The guidelines will be revised and refined on the basis of the experiences and discussions of this virtual workshop and the outcome of an intercomparison exercise organised as part of the project. This will be open to all internal dosimetry professionals.
\end{abstract}

Key words: guidelines / internal dose / IDEAS / harmonization

\title{
1. Introduction
}

La détermination des doses internes est une composante essentielle des programmes de surveillance individuelle des travailleurs. Elle peut aussi s'avérer nécessaire pour des personnes du public susceptibles d'incorporer des radionucléides dans le cadre de la médecine nucléaire ou de rejet accidentel de radionucléides dans l'environnement. L'évaluation des doses internes peut être divisée en deux phases, à savoir: (i) la détermination de la quantité de matière radioactive dans le corps humain, dans les organes ou dans une plaie par mesure directe et/ou par des méthodes indirectes comme l'analyse de l'excrétion ou la surveillance de l'air ambiant, et (ii) l'interprétation des données de surveillance en termes d'incorporation et/ou de dose interne en prenant en compte un grand nombre de facteurs et d'hypothèses concernant les caractéristiques physico-chimiques des substances radioactives, le mode d'incorporation, les processus biocinétiques et d'absorption énergétique, les paramètres individuels etc. La seconde phase est particulièrement délicate en raison du nombre de variables et de l'incertitude associée. Bien que la Commission internationale de protection radiologique (CIPR) et l'Agence internationale de l'énergie atomique (AIEA) aient publié des tables détaillées de coefficients de dose par unité d'incorporation (ICRP, 1998 ; IAEA, 1996), il s'agit de valeurs par défaut correspondant à des hypothèses sur différents paramètres qui peuvent ne pas être 
valides dans des situations spécifiques. La détermination de l'incorporation et de la dose interne qui en résulte peut donc être effectuée de plusieurs façons différentes, en fonction de la quantité et de la qualité des données, de la compétence du dosimétriste, des outils de calcul disponibles, et des hypothèses faites. Lorsqu'un même jeu de résultats de mesures est confié à deux dosimétristes différents, il est vraisemblable qu'il sera interprété de façons différentes sur la base de méthodes et de modèles dosimétriques différents et, en conséquence, que différentes solutions numériques seront obtenues. Ceci a été démontré par différents exercices d'intercomparaison, comme celui de l'AIEA (Andrasi et al., 1999) et la troisième intercomparaison européenne en calcul de dose interne (Doerfel et al., 2000).

La CIPR a récemment développé une nouvelle génération de modèles plus réalistes pour la dosimétrie interne. Ceux-ci incluent le modèle du tractus respiratoire humain [HRTM, publication 66 de la CIPR (ICRP, 1994)] et les modèles systémiques recyclants pour les actinides [publications 67 de la CIPR (ICRP, 1993) et 69 (ICRP, 1995)]. Ces modèles offrent une base pour prédire de façon réaliste l'excrétion et la rétention. Quelques recommandations simples ont été émises par la CIPR pour la surveillance individuelle dans les publications 54 et 78 de la CIPR (ICRP, 1998). Ces recommandations comprennent les indications générales suivantes :

- la surveillance de routine est conduite périodiquement dans les conditions normales d'activité. L'hypothèse de contamination retenue, en première intention, est celle d'une incorporation unique au milieu de l'intervalle de surveillance ;

- dans le cas d'une surveillance spéciale après un incident avéré ou l'accomplissement d'une tâche à risque, il est supposé qu'une incorporation aiguë s'est produite à la date correspondante ;

- la reconstruction d'une incorporation est, en général, effectuée à partir de la seule valeur de mesure réalisée à la fin de l'intervalle de surveillance considéré. Cependant, si plus de $10 \%$ de l'activité mesurée peut être attribuée à des incorporations dans des intervalles de surveillance antérieurs, une correction est recommandée pour prendre en compte cette contribution des incorporations précédentes ;

- en cas d'inhalation, tous les systèmes d'interprétation requièrent une information a priori sur le type d'absorption pulmonaire et sur la taille des particules composant l'aérosol contaminant. En l'absence d'information sur la taille des particules, il est recommandé de faire l'hypothèse d'une valeur par défaut du diamètre aérodynamique médian en activité (DAMA) de $5 \mu \mathrm{m}$.

Néanmoins, ces conseils laissent ouvert le choix de nombreuses hypothèses, résultant en autant d'interprétations différentes des données de mesure. Cette variabilité des interprétations a été illustrée par la troisième intercomparaison européenne de calcul en dosimétrie interne (Doerfel et al., 2000), qui s'est particulièrement intéressée aux effets des nouveaux modèles et au choix des paramètres d'entrée sur 
l'estimation des doses internes à partir des résultats de mesure de surveillance. Elle abordait aussi des questions qui ne l'avaient pas été dans les précédents exercices, comme la surveillance de l'air ambiant, les radioéléments naturels et l'exposition du public. Sept scénarios de contamination furent distribués qui concernaient les radio-isotopes ${ }^{3} \mathrm{H},{ }^{90} \mathrm{Sr},{ }^{125} \mathrm{I},{ }^{137} \mathrm{Cs},{ }^{210} \mathrm{Po},{ }^{238} \mathrm{U}$ et ${ }^{239} \mathrm{Pu}$. Les résultats de 50 participants furent collectés et ce fut, de loin, l'exercice de ce type le plus important à avoir été organisé jusqu'à maintenant. La plupart des participants ont traité plus de la moitié des cas. Ainsi, il y eut environ 35 réponses par cas pour un total d'environ 240 réponses. Les résultats en termes d'incorporation et de dose efficace engagée suivaient une distribution log-normale de déviation géométrique standard allant de 1,15 pour les cas impliquant $d{ }^{3} \mathrm{H}$ ou du ${ }^{137} \mathrm{Cs}$ à 2,4 pour les cas impliquant du ${ }^{239} \mathrm{Pu}$. Ces chiffres reflètent les larges divergences dans les résultats individuels qui varient, pour les cas extrêmes, dans une plage de cinq ordres de grandeur. Un élément clé de l'exercice fut un séminaire impliquant la plupart des participants, au cours duquel chaque cas et les approches utilisées pour l'évaluation furent discutés. Les raisons des différences entre les résultats ont été identifiées et incluaient des hypothèses différentes sur le profil temporel d'incorporation et sur le choix du modèle. La conclusion la plus importante de l'exercice fut la nécessité de développer des recommandations consensuelles pour les procédures d'évaluation de la dose interne afin de promouvoir l'harmonisation des évaluations entre les différentes organisations et les différents états. Cette harmonisation revêt une importance essentielle pour les pays membres de l'Union européenne.

À partir de ces éléments, le projet de recherche intitulé «General guidelines for the estimation of committed dose from incorporation monitoring data », d'acronyme «IDEAS », a été proposé et accepté par la Commission européenne dans le cinquième programme cadre pour la recherche et le développement technologique $(\mathrm{PCRD})^{10}$. Le projet IDEAS a débuté en octobre 2001 et sera achevé en mai 2005.

\footnotetext{
${ }^{10}$ Les institutions partenaires impliquées dans le projet sont :

1. Forschungzentrum Karlsruhe (FZK), Allemagne, co-ordinateur et responsable de la partie 4 du projet ;

2. Centre d'étude nucléaire $(\mathrm{SCK} \cdot \mathrm{CEN})$, Belgique, responsable de la partie 1 ;

3. Électricité de France (EdF), France ;

4. Agence nationale pour les nouvelles technologies, l'énergie et l'environnement (ENEA), Italie, responsable de la partie 3

5. Institut de radioprotection et de sûreté nucléaire (IRSN), France ;

6. Institut de recherche sur l'énergie atomique (AEKI), Hongrie, responsable de la partie 5 ;

7. Institut de radioprotection (RPI), Ukraine, responsable de la partie 2 ;

8. National Radiological Protection Board (NRPB), Royaume-Uni.

Le consortium, composé de représentants de ces huit organisations, s'est constitué sur la base de l'intérêt commun pour les problèmes abordés, de la complémentarité de l'expertise et des contacts établis au cours de collaborations précédentes. Son implication en calcul de dose interne résulte de différentes démarches. Dans la plupart des cas, elle complémente principalement la surveillance par mesure in vivo et in vitro (EdF, ENEA, FZK, AEKI, SCK•CEN). Néanmoins, dans d'autres cas, elle est principalement liée à l'implication dans le développement de modèles utilisés pour relier l'incorporation de radioéléments aux doses aux organes et à l'excrétion (IRSN, NRPB), et/ou au développement de logiciels pour implémenter des modèles et assurent différentes fonctions : instituts de recherche et/ou d'étude (ENEA, FZK, AEKI, SCK •CEN, IRSN), autorité nationale de radioprotection (NRPB, RPI) et production d'énergie nucléaire (EdF), et combinent ainsi des perspectives différentes.
} 
Partie 1 : création d'une base de données de cas de contamination Partenaires 1, 2, 3, 4, 5, 6, 7, 8
Partie 2 : développement du logiciel d'évaluation

Partenaires 1, 7, 8

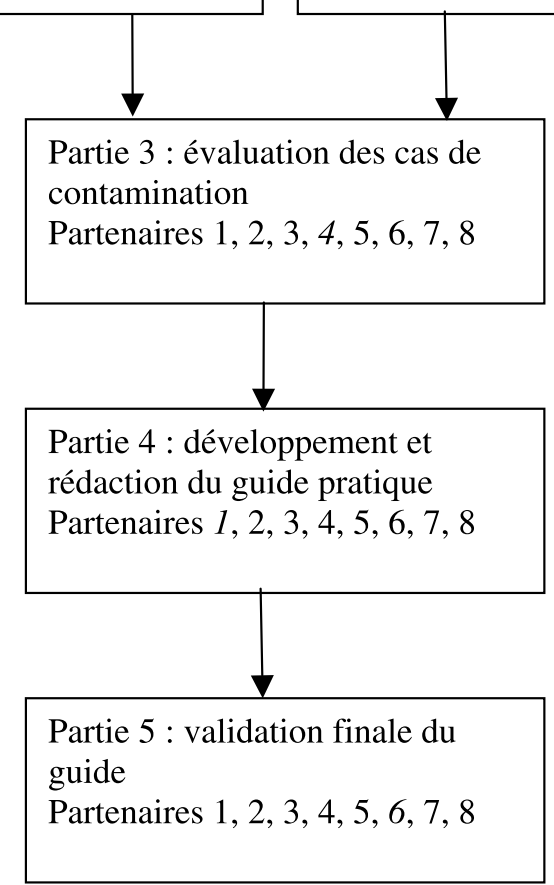

Figure 1 - Structure du projet (les animateurs des différentes parties sont indiqués par des numéros en italique).

Structure of Work Packages (Work Package leaders are indicated in italic numbers).

\section{Programme de travail}

Le projet IDEAS est divisé en parties (Work Packages) correspondant aux cinq objectifs les plus importants. La structure du projet et l'interaction entre les parties sont représentées en figure 1.

La première partie fut dédiée à la collecte de données par le biais de recherches bibliographiques dans la littérature ouverte, de l'obtention de données auprès d'organismes spécifiques et en utilisant l'information des bases de données de cas de contamination existantes. Deux bases de données (la base de données 
bibliographiques et la base de données des cas de contamination) ont ainsi été créées et des cas de référence ont été sélectionnés pour une évaluation croisée. Simultanément, un logiciel existant a été amélioré à l'aide d'un nouvel algorithme d'interprétation des données et de nouvelles méthodes d'interprétation des données ont été étudiées et comparées. Les cas de référence sélectionnés ont ensuite été évalués par au moins deux partenaires à l'aide du même logiciel. Les évaluations ont été compilées en une base de données et analysées pour mettre en évidence les hypothèses communément adoptées pour des scénarios similaires, les modèles et les paramètres utilisés, ainsi que les procédures suivies pour estimer les incertitudes, gérer les données aberrantes et les résultats de mesure sous la limite de détection. Enfin, les partenaires ont tiré de l'évaluation des données de surveillance une stratégie commune, rédigé une première version du guide et l'ont discutée avec les experts de la dosimétrie interne au cours d'un premier séminaire, tenu sur Internet. Cette discussion a permis d'améliorer la stratégie commune et de finaliser les conseils pratiques.

Un exercice d'intercomparaison en calcul de dose ouvert aux experts du monde entier (4th European Intercomparison Exercise on Internal Dose Assessment) offrira un test de la validité du guide nouvellement rédigé. L'intercomparaison sera préparée conjointement avec l'AIEA, tous les participants recevront le guide et seront invités à l'utiliser pour l'évaluation des cas de contamination. L'organisation d'un second séminaire, ouvert à tous les participants de l'intercomparaison, pour discuter des résultats et finaliser le rapport sur l'intercomparaison est aussi prévue. La dernière étape sera la publication de la version finale $\mathrm{du}$ guide et sa soumission aux organismes nationaux et internationaux pour approbation.

\section{3. État d'avancement du projet}

À la date de juillet 2004, deux bases de données ont été construites. La première, intitulée base de données bibliographiques (BibDb), rassemble les données présentes dans la littérature ouverte ou dans des rapports traitant de cas de contamination. Sa structure permet à l'utilisateur de la visualiser, d'y faire des recherches et d'entrer de nouvelles données. Jusque là, plus de 500 références ont été rassemblées. À côté de son utilisation dans le cadre du projet IDEAS, BibDb constitue aussi un outil utile à la communauté scientifique de la dosimétrie interne pour l'étude de cas de contamination. Les descriptions de cas sélectionnés pour leur intérêt pour l'évaluation de la dose interne ont été rassemblées dans une autre base de données appelée base de données de contamination interne (IntContDb). Cette seconde base de données a été conçue pour rassembler, à un format donné, les descriptions des cas de contamination sélectionnés (scénarios de contamination 
et mesures de suivi). La structure d'IntContDb permet de rassembler toutes les informations nécessaires à l'estimation de la dose, c'est-à-dire la description du lieu de travail et les caractéristiques de ce travail, la date et les conditions de l'événement de départ, les actions entreprises, les caractéristiques physicochimiques du contaminant, etc. Cette base de données contient actuellement plus de 200 cas. Pour le moment, IntContDb et BibDb ne sont accessibles qu'aux partenaires d'IDEAS, mais seront ultérieurement accessibles au public sur Internet.

Le code de calcul IMIE [Individual Monitoring of the Internal Exposure (Berkovski, 2000)] a été choisi puis amélioré et ajusté pour l'évaluation des cas de référence sélectionnés. Il donne au dosimétriste un très bon outil pour l'analyse et l'interprétation de mesures multiples d'échantillons biologiques en aidant l'expert à faire des estimations de l'historique de contamination et des doses correspondantes sur la base des données de surveillance individuelle. Il permet en particulier à l'utilisateur de passer en revue et de comparer les combinaisons de paramètres d'exposition envisageables. Parmi ces paramètres, la prise en compte d'un éventuel traitement de décorporation s'avère particulièrement difficile à formaliser et le sujet est proposé pour discussion à la communauté scientifique de dosimétrie interne.

Soixante sept cas couvrant différentes circonstances et seize radionucléides ont été sélectionnés et évalués en utilisant les codes IMIE (Berkovski, 2000) et IMBAexpert (Birchall et al., 2003), en faisant différentes hypothèses. La meilleure estimation calculée de l'incorporation et de la dose efficace engagée a été donnée pour chaque cas, conjointement avec des notes sur les questions importantes pour la rédaction du guide. Les résultats ont été rassemblés dans une base de données des évaluations (EvalDb) reliée à l'IntContDb. Jusqu'à maintenant, 75 évaluations indépendantes de 42 cas ont été incluses dans cette base de données. Les résultats de l'évaluation des cas ont été discutés par les partenaires sous différents aspects (ajustement du modèle aux données, incertitude sur les données, profil d'incorporation et paramètres du modèle, type de surveillance préférentielle, gestion des données de surveillance, etc.) et les leçons ont été retirées en vue de la préparation du guide.

La première ébauche du guide d'IDEAS été harmonisée avec le document CIPR correspondant en préparation (Fry et al., 2003). Cette version préliminaire est soumise aux commentaires d'autant d'experts de la dosimétrie que possible à travers le monde, par le moyen d'un « séminaire virtuel » sur Internet. Les versions successives du guide et les bases de données établies dans le cadre du projet IDEAS seront mises à disposition sur le site Internet du projet IDEAS, en accès libre à l'adresse http://www.ideas-workshop.de. Les commentaires sont les 
bienvenus et seront affichés, ainsi que leurs réponses sur ce site Internet. Les premiers commentaires ont été discutés par le consortium et une version révisée du guide pratique est en cours de préparation qui prend en compte l'apport du « séminaire virtuel ». La validation du guide sera faite à travers un exercice d'intercomparaison organisé conjointement avec l'AIEA, prévu pour être achevé en mars 2005 et suivi par un autre séminaire afin de tirer les conclusions finales sur l'utilisation de ces conseils et de permettre la rédaction de leur version définitive.

\section{Coopération avec d'autres projets}

Une collaboration étroite avec d'autres groupes internationaux ayant des intérêts communs avec IDEAS est prévue par le programme du projet, elle inclut :

- le projet européen OMINEX, qui s'intéresse à l'optimisation des procédures permettant d'obtenir les données de surveillance de l'incorporation (Etherington et al., 2003) ;

- le projet européen IDEA, qui s'occupe principalement de l'amélioration des méthodologies de surveillance directe et indirecte afin d'augmenter la sensibilité de détection ;

- le projet européen BIODOS, qui vise une meilleure compréhension du comportement des radionucléides dans le corps humain et

- le groupe de travail CIPR sur l'interprétation des données biologiques, qui vise la préparation d'un document technique de la CIPR sur la dosimétrie rétrospective (Fry et al., 2003).

L'échange de l'information et la participation croisée des participants des projets enrichissent le travail d'IDEAS.

\section{Diffusion et utilisation des résultats}

La diffusion des résultats fait partie intégrante du projet. Trois groupes d'utilisateurs potentiels des recommandations sont pris en compte :

- les médecins du travail qui réalisent la surveillance de routine et la surveillance spéciale des travailleurs afin d'estimer leurs doses internes dues soit à la manipulation journalière de matière radioactive, soit à des incidents ou accidents de contamination ;

- des groupes d'experts impliqués dans le développement de recommandations ou de références concernant l'exposition aux matières radioactives, en particulier les membres du groupe de travail CIPR sur la dosimétrie interne (INDOS) qui développent des révisions des publications 30/68 et 54/78 de la CIPR ; 
- les autorités nationales et internationales qui normalisent et contrôlent les procédures de radioprotection.

Les utilisateurs potentiels sont informés du projet et de l'évolution du guide pratique par des contacts personnels, à travers des conférences et des réunions, et via l'Internet.

Remerciements. Ce projet est financé en partie par la Commission européenne à travers le contrat $N^{\circ}$ FIKR-CT2001-00160. Les auteurs souhaitent manifester leur reconnaissance pour le soutien de la Commission.

\section{RÉFÉRENCES}

Andrasi A., Doerfel H., Hui E., Ouvrard R. (1999) Intercomparison and Biokinetik Model Validation of Radionuclide Intake Assessment, International Atomic Energy Agency, IAEA-TECDOC1071.

Berkovski V. (2000) Application of the internal dosimetry support system for interpretation of in vivo and bioassay measurements, Radiat. Prot. Dosim. 89, 271.

Birchall A., Puncher M., Marsh J.W., et al. (2003) IMBA-EXPERT: internal dosimetry made simple. Presented at the Workshop on Internal Dosimetry of Radionuclides: Occupational, Public and Medical Exposure, Oxford, 9-12 September 2002, Radiat. Prot. Dosim. 105, 421-425.

Doerfel H., Andrasi A., Bailey M.R., Birchall A., Castellani C.-M., Hurtgen C., Jarvis N., Johansson L., Leguen B., Tarroni G. (2000) Third European Intercomparison Exercise on Internal Dose Assessment, Research Center Karlsruhe, Research Report FZKA 6457, Karlsruhe, ISSN 09478620.

Etherington G., Stradling G.N., Rahola T., et al. (2003) Design and implementation of monitoring programmes for internal exposure (Project OMINEX). Presented at the Workshop on Internal Dosimetry of Radionuclides: Occupational, Public and Medical Exposure, 9-12 September 2002, Oxford, Radiat. Prot. Dosim. 105, 641-644.

Fry F.A., Lipsztein J.L., Birchall A. (2003) The ICRP Working Party on Bioassay Interpretation. Presented at the Workshop on Internal Dosimetry of Radionuclides: Occupational, Public and Medical Exposure, Oxford, 9-12 September 2002, Radiat. Prot. Dosim. 105, 297-302.

IAEA (1996) Safety Series No. 115, International atomic energy agency, food and agriculture organization of the united nations, international labour organisation, nuclear energy agency of the organization for economic co-operation and development, pan american health organization, world health organization: International basic safety standards for protection against ionizing radiation and for the safety of radiation sources, IAEA, Vienna.

ICRP Publication 66 (1994) Human Respiratory Tract Model for Radiological Protection, Ann. ICRP 24.

ICRP Publication 67 (1993) Age-dependent Doses to Members of the Public from Intakes of Radionuclides: Part 2. Ingestion Dose Coefficients, Ann. ICRP 23.

ICRP Publication 69 (1995) Age-dependent Doses to Members of the Public from Intakes of Radionuclides: Part 3. Ingestion Dose Coefficients, Ann. ICRP 25.

ICRP Publication 78 (1998) Individual Monitoring for Internal Exposure of Workers, Ann. ICRP 27. 